GEOMETRY IN NONLINEAR CONTROL

AND DIFFERENTIAL INCLUSIONS

BANACH CENTER PUBLICATIONS, VOLUME 32

INSTITUTE OF MATHEMATICS

POLISH ACADEMY OF SCIENCES

WARSZAWA 1995

\title{
SINGULARITIES AND NORMAL FORMS OF SMOOTH DISTRIBUTIONS
}

\author{
M. ZHITOMIRSKII \\ Department of Mathematics, Technion, 32000 Haifa, Israel
}

\begin{abstract}
In this expository paper we present main results (from classical to recent) on local classification of smooth distributions.
\end{abstract}

0. Introduction. This paper is devoted to the classification of germs of smooth generic $k$-distributions on an $n$-manifold $M^{n}$ ( $k$-dimensional subbundles of the tangent bundle $T M$ ) with respect to the natural equivalence (section 1). We assume that $k \geq 2$ (if $k=1$ then we deal with fields of lines; all fields of lines are locally equivalent).

The first arising question is the following: is the codimension of the orbit of the germ at a generic point of a generic $k$-distribution on $M^{n}$

a) 0 (in this case the germ is stable and reducible to a model, i.e. a normal form without parameters),

b) finite (in this case the germ is finitely determined and reducible to a polynomial normal form; it might be both stable and unstable),

c) infinite (in this case functional moduli appear, and the germ is always unstable).

It turns out that for generic germs the answer is either a) or c) depending on the pair $(k, n)$. The answer is a) if and only if $k=n-1$ or $k=2, n=4$. In the first case all generic germs are equivalent to the Darboux-Pfaff model. The second case is also classical, but not so well-known. The equivalence of all generic germs of 2-distributions on $M^{4}$ was proved by Engel 100 years ago. The Darboux and Engel models are given in section 2 .

If $2 \leq k \leq n-2$ and $(k, n) \neq(2,4)$ then functional moduli appear: though it seems that the latter was known at the beginning of the century the proof

1991 Mathematics Subject Classification: 58A30.

The work on this paper was supported in part by Technion VPR Fund.

The paper is in final form and no version of it will be published elsewhere. 
appeared only recently (B. Jakubczyk and F. Przytycki (1984); A. Vershik and V. Gershkovich (1988)). The proof is based on simple "dimensional arguments" and we repeat it in section 3. Asymptotically exact formal normal forms (normal forms on the level of formal series containing the "right number" of functional parameters) are given in section 4. They were obtained in the author's paper, 1991. Recently it was proved that the same normal forms hold for 2-distributions (R. Bryant and L. Hsu (1993)) and for 3-distributions on $M^{5}$ (R. Bryant (1994)).

If the initial problem is the classification of distributions of some fixed structure then a generic germ might be stable for all $(k, n)$. To each distribution one can associate the growth vector (at any point). The growth vector of a generic $k$-distribution on $M^{n}$ is a fixed sequence depending on $k$ and $n$ only (section 5). One can fix a different growth vector and consider the space of distributions on $M^{n}$ with this growth vector at every point. Distributions whose growth vector is the same at all points are called regular. The simplest example of a regular distribution is an involutive $k$-distribution (the growth vector is $(k, k, \ldots))$. By the Frobenius theorem all involutive $k$-distributions are locally equivalent. It turns out that the same is true for regular distributions whose growth vector belongs to a certain set. Classification results for regular distributions were obtained by A. Vershik and V. Gershkovich (1989), by A. Kumpera and C. Ruiz (1982), and by the author (1990); we present some of them in section 6 .

Another direction in the classification of distributions is related to the singularities of fields of hyperplanes. Given a generic $(n-1)$-distribution $D$ on $M^{n}$ denote by $S$ the subset of $M^{n}$ consisting of points at which the germ of $D$ is not generic, i.e. is not equivalent to the Darboux model (this means that the Darboux genericity conditions are violated). The first who considered germs at points of $S$ was J. Martinet (1970). Martinet showed that

1) $S$ is a submanifold (for large $n$ it can be stratified);

2) $\operatorname{codim} S=1$ if $n$ is odd, and $\operatorname{codim} S=3$ if $n$ is even;

3 ) if $n$ is odd then the germ of $D$ at a generic point of $S$ is reducible to a normal form without parameters (Martinet model).

In the even-dimensional case there is at least one numerical invariant (Mormul (1988)). Normal forms of germs of $(2 k-1)$-distributions on $M^{2 k}$ at generic points of $S$ were obtained by the author $(1988,1992)$.

In the 3-dimensional case $S$ is a surface, and Martinet genericity conditions are violated at isolated points of $S$. Martinet conjectured that the germ of $D$ at each of these isolated points is stable, but B. Jakubczyk and F. Przytycki showed (1979) that this is not true: there exists at least one numerical invariant. Later the author proved $(1989,1992)$ that there are no other invariants and obtained a polynomial normal form. So, the classification of typical singularities of fields of planes on $M^{3}$ is completed. Main results are given in section 8 .

In sections 7 and 9 we present classification results and geometry of singularities for fields of hyperplanes on $M^{n}, n \geq 4$. A complete list of stable germs and 
a complete list of finitely determined typical singularities were obtained in the author's works $(1989,1990,1992)$.

Results concerning singularities of 2-distributions on $M^{4}$ were announced in the author's paper (1990a). We present them in section 10. The set of points at which the germ of a generic distribution is not equivalent to the Engel model is the union of two 2-dimensional surfaces; these surfaces are not transversal and their intersection is a curve. One of the surfaces contains another curve invariantly related to the distribution; the germ at any point outside the curves is stable; classification of the other germs contains functional moduli.

Sections 11 and 12 contain complete lists of all stable germs and all finitely determined germs of $k$-distributions on $M^{n}$, for all $k$ and $n$.

The classification results for smooth distributions have a number of applications for control theory, PDE's, contact geometry. Recently the normal forms were fruitfully applied to the problems of the sub-Riemannian geometry (R. Montgomery (1993), R. Bryant and L. Hsu (1993), W. Liu and H. Sussmann (1994)).

1. Local equivalence of smooth distributions. All objects considered below are assumed to be smooth (of class $C^{\infty}$ ).

1.1. A smooth $k$-distribution on an $n$-dimensional manifold $M^{n}$ is a smooth $k$ dimensional subbundle of the tangent bundle, i.e. a family $\left\{\Sigma(\alpha) \subset T_{\alpha} M^{n}\right\}_{\alpha \in M^{n}}$, where the subspace $\Sigma(\alpha)$ depends smoothly on the point $\alpha$.

1.2. For example, a 1-distribution is a field of directions, an $(n-1)$-distribution is a field of hyperplanes.

1.3. A $k$-distribution $\Sigma$ on $M^{n}$ can be given (at least locally) as a $k$-generated module $\Sigma=V=\left(v_{1}, \ldots, v_{k}\right)$ of vector fields (over the ring of smooth functions) or as an $(n-k)$-generated module $\Sigma=W=\left(w_{1}, \ldots, w_{n-k}\right)$ of differential 1-forms (Pfaffian system). Then $\Sigma(\alpha)=V(\alpha)$ or $\Sigma(\alpha)=W(\alpha)$ where $V(\alpha)$ is a subspace generated by the tangent vectors $v_{1}(\alpha), \ldots, v_{k}(\alpha)$, and $W(\alpha)$ is the subspace of tangent vectors annihilated by the functionals $\left.w_{1}\right|_{\alpha}, \ldots,\left.w_{n-k}\right|_{\alpha}$.

1.4. In order to classify distributions we have to find a suitable coordinate system and suitable generators of the module $\left(v_{1}, \ldots, v_{k}\right)$ or $\left(\omega_{1}, \ldots, \omega_{n-k}\right)$ such that the generators have form as simple as possible. This problem has a number of applications (for control theory, partial differential equations, non-holonomic dynamics, contact geometry).

1.5. It follows from 1.3 that a precise definition of local equivalence of distributions should involve local equivalence of modules of vector fields or differential 1-forms. We call two germs of modules $V$ and $\tilde{V}$ of vector fields equivalent if there exists a local diffeomorphism $\Phi$ such that $\Phi_{*} V=\tilde{V}$. The latter equality means that $\Phi$ carries the germ of any vector field from $V$ to the germ of some vector field from $\tilde{V}$, and $\Phi^{-1}$ carries the germ of any vector field from $\tilde{V}$ to the germ of 
some vector field from $V$. The definition of local equivalence of Pfaffian systems (modules of differential 1-forms) is similar.

1.6. There exists another, equivalent definition of the local equivalence of modules of vector fields or Pfaffian systems which is more convenient for applications. Two local $r$-generated modules $\left(g_{1}, \ldots, g_{r}\right)$ and $\left(\tilde{g}_{1}, \ldots, \tilde{g}_{r}\right)$ are called equivalent if there exists a local diffeomorphism $\Phi$ and a nondegenerate $r \times r$ matrix valued function $H$ such that

$$
\Phi .\left[g_{1}, \ldots, g_{r}\right]=\left[\tilde{g}_{1}, \ldots, \tilde{g}_{r}\right] H
$$

(equality of two tuples). Here $\Phi_{.}=\Phi_{*}$ for modules of vector fields, $\Phi_{\text {. }}=\Phi^{*}$ for Pfaffian systems, $\Phi .\left[g_{1}, \ldots, g_{r}\right]=\left[\Phi . g_{1}, . ., \Phi . g_{r}\right]$.

This definition is independent of the choice of the generators of the modules and is equivalent to the definition of 1.5 , even if the dimension of distributions is not constant (Jakubczyk, Przytycki (1984): Appendix).

1.7. For example, two local $(n-1)$-distributions (fields of planes) $(\omega)$ and $(\tilde{\omega})$ are equivalent if and only if there exists a local diffeomorphism $\Phi$ and a germ $H$ of a nonvanishing function such that $\Phi^{*} \omega=H \tilde{\omega}$.

\section{Classical classification results}

2.1. The simplest classification holds in the case $k=1$ (fields of directions). A 1-distribution is generated, in suitable coordinates, by the vector field $\frac{\partial}{\partial x_{1}}$ (this follows from the basic theorem on systems of ODE's).

2.2. The case $k=n-1$ (fields of hyperplanes) is also classical, but it is much more difficult. It follows from Darboux theorems on classification of differential 1-forms that a generic germ $\left(^{1}\right)$ of a field of hyperplanes on $M^{n}$ is equivalent to the Pfaffian equation

$$
\begin{array}{ll}
W=\left(d z+x_{1} d y_{1}+\ldots+x_{k} d y_{k}\right) & (n=2 k+1), \\
W=\left(d y_{1}+x_{2} d y_{2}+\ldots+x_{k} d y_{k}\right) & (n=2 k)
\end{array}
$$

(in suitable coordinates $z, x_{1}, y_{1}, \ldots, x_{k}, y_{k}$ if $n=2 k+1$ and $x_{1}, y_{1}, \ldots, x_{k}, y_{k}$ if $n=2 k$ )

2.3. Distribution (2.1) (resp. (2.2)) is called the standard contact (resp. quasicontact) structure. The germ at a generic point of a generic globally defined field of hyperplanes on an odd- (resp. even-) dimensional manifold is equivalent to (2.1) (resp. (2.2)).

2.4. There exists one more classical case investigated by Engel (Engel, 1889): the case $k=2, n=4$. Engel proved that a generic germ of 2-distribution on $R^{4}$

$\left({ }^{1}\right)$ We say that a statement holds for a generic germ if there exists an integer $l$ and an open dense subset $Q$ of the space of $l$-jets such that the statement is true for all germs whose $l$-jet belongs to $Q$. The genericity conditions under which a germ is reducible to one of the normal forms below are given in sections $7-10$. 
is equivalent to

$$
V=\left(\frac{\partial}{\partial x_{2}}, \frac{\partial}{\partial x_{1}}+x_{3} \frac{\partial}{\partial x_{4}}+x_{2} \frac{\partial}{\partial x_{3}}\right)
$$

or, in terms of Pfaffian systems,

$$
W=\left(d x_{4}-x_{3} d x_{1}, d x_{3}-x_{2} d x_{1}\right) .
$$

3. Functional moduli in the non-classical cases

(Jakubczyk and Przytycki (1984); Vershik and Gershkovich (1988))

3.1. By the non-classical cases we mean the local classification problem for $k$-distributions on $M^{n}$, where $2 \leq k \leq n-2$, and $(k, n) \neq(2,4)$. In the classical cases normal forms of generic germs are simple (contain no parameters); in the non-classical cases it is not so, and, moreover, functional moduli appear even in the classification of generic germs. This means that the orbit (equivalence class) of a generic germ has infinite codimension in the space of all germs. The arguments showing this are as follows.

3.2. To give a germ of a $k$-generated module of vector fields on $R^{n}$ in local coordinates one has to present $k n$ functions in $n$ variables. This means that the functional dimension $d_{1}$ of the space of all $k$-generated modules of vector fields on $R^{n}$ is equal to $k n$. Two modules are equivalent if the corresponding tuples of generators are equivalent with respect to the action of the product of two groups - the group of changes of coordinates and the group of nondegenerate $k \times k$ matrix valued functions (see 1.6). A change of coordinates is defined by $n$ functions in $n$ variables, therefore the functional dimension $d_{2}$ of the group of all possible transformations is equal to $k^{2}+n$. Simple arguments (based on the fact that local classification of germs includes algebraic classification of jets of any finite order) show that the codimension of any germ's orbit is infinite if $d_{2}<d_{1}$, i.e. $k^{2}+n<k n$. It is easy to see that this inequality holds true for all the pairs $(k, n)$ such that $k \leq n$ except the classical cases $k=1, k=n-1$ and $k=2, n=4$.

\section{Normal forms of generic germs in the non-classical cases}

(Zhitomirskiı (1991); Bryant and Hsu (1993); Bryant (1994))

4.1. The attempt to obtain a complete classification of generic germs for the non-classical pairs $(k, n)$ seems to be hopeless. On the other hand, it is possible to obtain asymptotically exact normal forms, which are valuable for applications. By an asymptotically exact normal form we mean a family $N$ of germs, parametrized by a tuple of functions in $n$ variables, such that a generic germ of a distribution is formally (i.e. on the level of formal series) equivalent to some (not unique) germ of $N$, and the number $p_{k}$ of numerical parameters of the family $j^{k} N$ ( $k$-jets of the germs from $N$ ) grows asymptotically (as $k \rightarrow \infty$ ) in the same way as the number of modules $m_{k}$ appearing in the classification of the $k$-jets of generic germs, i.e. $p_{k} / m_{k}=1+o(1)$ as $k \rightarrow \infty$. An asymptotically exact normal form $N$ cannot 
be essentially simplified (though two germs from $N$ might be equivalent). Using the arguments of section 3 one can conclude that a family $N$ is an asymptotically exact normal form if any germ is equivalent to some germ of $N$, and the elements of $N$ are parametrized by a tuple of $k n-n-k^{2}$ functions in $n$ variables.

4.2 (Zhitomirskiı (1991)). Asymptotically exact normal forms of generic germs of $k$-distributions on $M^{n}$ are as follows. Let $p=n-k$ (the codimension of a distribution), $s=[k / 2]$. Define

$$
\begin{aligned}
& \omega_{1}=d x_{1}+\sum_{j=1}^{s} x_{p+2 j-1} d x_{p+2 j} \\
& \omega_{2}=d x_{2}+\sum_{j=p+3}^{n} f_{2, j}(x) d x_{j} \quad \text { if } k \geq 3 \\
& \omega_{2}=d x_{2}+x_{n-1}^{2} d x_{p+2}+\sum_{j=p+3}^{n} f_{2, j}(x) d x_{j} \quad \text { if } k=2, \\
& \omega_{m}=d x_{m}+\sum_{j=p+2}^{n} f_{m, j}(x) d x_{j}, \quad m=3, \ldots, p
\end{aligned}
$$

Then the family of Pfaffian systems $\left(\omega_{1}, \ldots, \omega_{p}\right)$, with the functional parameters

$$
f_{2, p+3}, \ldots, f_{2, n}, f_{3, p+2}, \ldots, f_{3, n}, \ldots, f_{p, p+2}, \ldots, f_{p, n}
$$

is an asymptotically exact normal form of generic germs of $k$-distributions on $M^{n}$.

4.3. Consider a particular case of 2 -distributions. In this case it is more convenient to give an asymptotically exact normal form in terms of vector fields. From normal form in 4.2 one can pass to the normal form

$$
\left(\frac{\partial}{\partial x_{1}}, \frac{\partial}{\partial x_{2}}-x_{1} \frac{\partial}{\partial x_{3}}-x_{1}^{2} \frac{\partial}{\partial x_{4}}-f_{1}(x) \frac{\partial}{\partial x_{5}}-\ldots-f_{n-4}(x) \frac{\partial}{\partial x_{n}}\right)
$$

with functional parameters $f_{1}, \ldots, f_{n-4}$.

If $n=4$ then this normal form contains no parameters and is equivalent to Engel's normal form given in 2.4.

4.4. Consider another particular case of $(2 n-1)$-distributions on $R^{2 n+1}$, $n \geq 2$. In this case the normal form in 4.2 is as follows:

$$
\begin{aligned}
\left(d x_{1}+x_{3} d x_{4}+x_{5} d x_{6}+\ldots+x_{n-2} d x_{n-1},\right. & \\
& \left.d x_{2}+f_{1}(x) d x_{5}+f_{2}(x) d x_{6}+\ldots+f_{n-4}(x) d x_{n}\right)
\end{aligned}
$$

if $n$ is odd, and

$$
\left(d x_{1}+x_{3} d x_{4}+x_{5} d x_{6}+\ldots+x_{n-1} d x_{n}, d x_{2}+f_{1}(x) d x_{5}+f_{2}(x) d x_{6}+\ldots+f_{n-4}(x) d x_{n}\right)
$$

if $n$ is even. 
4.5. One more example: $k=3, n=5$. In this case the normal form contains one functional parameter, and a distribution is equivalent to the Pfaffian system

$$
\left(d x_{1}+x_{3} d x_{4}, d x_{2}+f(x) d x_{5}\right) .
$$

4.6. Conjecturally, a generic germ is equivalent to a normal form given in 4.2 not only formally, but also smoothly. This is proved by R. Bryant and L. Hsu for 2-distributions (1993); answering the author's question R. Bryant also proved that a generic germ of a 3-distribution on $M^{5}$ is smoothly equivalent to the normal form given in 4.5 .

\section{Growth vector. Regular distributions}

5.1. Classification of distributions of some fixed structure might contain no functional moduli. For example, any involutive $k$-distribution (i.e. a module of vector fields which is a Lie algebra) is locally equivalent to $\left(\frac{\partial}{\partial x_{1}}, \ldots, \frac{\partial}{\partial x_{k}}\right)$.

5.2. We will consider distributions of more general structure: regular distributions with a fixed growth vector (the involutive case is the case of trivial growth vector).

Let $V=\left(v_{1}, \ldots, v_{k}\right)$ be a module of local vector fields on $M^{n}$. Let $V_{1}=V$, and $V_{2}$ be the minimal module containing all vector fields from $V_{1}$ and all their Lie brackets (for example, if $k=2$ then $V_{2}$ is generated by $v_{1}, v_{2}$ and $\left[v_{1}, v_{2}\right]$ ). We will write $V_{2}$ in the form $V_{2}=\left[V_{1}, V_{1}\right]$. Define now $V_{3}=\left[V_{2}, V_{1}\right]$, i.e. $V_{3}$ is a minimal module of vector fields containing the fields from $V_{1}, V_{2}$ and all the Lie brackets $[v, \mu], v \in V_{1}, \mu \in V_{2}$. Define in the same way $V_{i}=\left[V_{i-1}, V_{1}\right], i=4,5, \ldots$ We have a sequence of modules $V_{1} \subset V_{2} \subset V_{3} \subset \ldots$ The sequence $a_{i}(\alpha)=\operatorname{dim} V_{i}(\alpha)$ is called the growth vector of $V$ at the point $\alpha$. A distribution $V$ is called regular if all the modules $V_{i}$ define distributions, i.e. $a_{i}(\alpha)$ does not depend on a point $\alpha \in M^{n}$ (examples below show that this is not always true even for generic distributions on $M^{n}$ ).

The sequence $a_{i}=\operatorname{dim} V_{i}$ stabilizes for any regular distribution $V$ (there exists $m$ such that $\left.a_{m}=a_{m+1}\right)$. The growth vector of a regular distribution is denoted by $\left(a_{1}=k, a_{2}, \ldots, a_{m}\right)$. If $a_{m}=n$ then the distribution is called non-holonomic or bracket generating.

5.3. A generic germ of a $k$-distribution in $R^{n}$ is regular and bracket generating. It has a so-called maximal growth vector (it depends just on $k$ and $n)$. If $(k, n)=$ $(2,6)$ then the growth vector is $(2,3,5,6)$. If $(k, n)=(2,9)$ then the growth vector is $(2,3,5,8,9)$.

5.4. Let us consider some examples of 2-distributions on $R^{4}$. The distribution (2.3) has the growth vector $(2,3,4)$ at every point of $R^{4}$, therefore it is regular. The distribution

$$
\left(v_{1}=\frac{\partial}{\partial x_{3}}, v_{2}=\frac{\partial}{\partial x_{4}}-x_{3}^{2} \frac{\partial}{\partial x_{1}}-x_{3} x_{4} \frac{\partial}{\partial x_{2}}\right)
$$


has the growth vector $(2,3,4)$ at a generic point of $R^{4}\left(x_{3} \neq 0\right.$ or $\left.x_{4} \neq 0\right)$, and the growth vector $(2,2,4)$ at points of the plane $x_{3}=x_{4}=0$.

The distributions

$$
\left(v_{1}=\frac{\partial}{\partial x_{3}}, v_{2}=\frac{\partial}{\partial x_{4}}-x_{3} \frac{\partial}{\partial x_{1}}-x_{3}^{2} x_{4} \frac{\partial}{\partial x_{2}}\right)
$$

and

$$
\left(v_{1}=\frac{\partial}{\partial x_{3}}, v_{2}=\frac{\partial}{\partial x_{4}}-x_{3} \frac{\partial}{\partial x_{1}}-\left(x_{3}^{3} / 3+x_{3} x_{4}^{2}\right) \frac{\partial}{\partial x_{2}}\right)
$$

have the growth vector $(2,3,4)$ at a generic point of $R^{4}\left(x_{3} \neq 0\right.$ or $\left.x_{4} \neq 0\right)$, and the growth vector $(2,3,3,4)$ at points of the plane $x_{3}=x_{4}=0$.

Therefore distributions (5.1)-(5.3) are not regular. Nevertheless, they correspond to typical singularities of 2-distributions on $M^{4}$ (see section 10).

\section{Classification results for regular distributions}

6.1 (Zhitomirskiu (1990)). Let $W_{A}$ be the set of germs of all regular completely non-holonomic $k$-distributions $(k \geq 2)$ on $M^{n}$ having the growth vector $A$ (the first coordinate of $A$ is equal to $k$, the last one is equal to $n$ ). There are three types of the growth vector $A$ such that the classification of generic germs within $W_{A}$ contains no functional moduli. They are as follows.

1) $A=(n-1, n)$. It follows from the Darboux theorem that a generic germ of $D_{A}$ is equivalent to $(2.1)$ or $(2.2)$.

2) $A=(n-2, n-1, n), n \geq 4$. All germs from $D_{(n-2, n-1, n)}$ are equivalent to the germ

$$
\left(\frac{\partial}{\partial x_{2}}, \frac{\partial}{\partial x_{1}}+x_{3} \frac{\partial}{\partial x_{4}}+x_{2} \frac{\partial}{\partial x_{3}}, \frac{\partial}{\partial y_{1}}, \ldots, \frac{\partial}{\partial y_{n-4}}\right) .
$$

3) $A=(n-3, n-2, n-1, n), n \geq 5$. All germs from $D_{(n-3, n-2, n-1, n)}$ are equivalent to the germ

$$
\left(\frac{\partial}{\partial x_{2}}, \frac{\partial}{\partial x_{1}}+x_{4} \frac{\partial}{\partial x_{5}}+x_{3} \frac{\partial}{\partial x_{4}}+x_{2} \frac{\partial}{\partial x_{3}}, \frac{\partial}{\partial y_{1}}, \ldots, \frac{\partial}{\partial y_{n-5}}\right) .
$$

6.2 (Vershik and Gershkovich (1989), Zhitomirskiŭ (1990)). Classification of germs of regular distributions having a fixed growth vector of any other type contains functional moduli.

6.3. For regular distributions on $R^{3}$ and $R^{4}$ we can formulate classification results stronger than in 6.1. If $n=3$ or $n=4$ then all germs of regular bracket generating $k$-distributions on $M^{n}$ are equivalent (the pair $(k, n)$ is the only invariant) to the germ $d z+x d y(k=2, n=3)$, or $d x_{1}+x_{2} d y_{2}(k=3, n=4$, the coordinate $x_{1}$ does not occur), or to the germ $(2.3)(k=2, n=4)$. This follows from genericity conditions under which a germ is equivalent to Darboux or Engel normal forms (we discuss the genericity conditions in sections $7-10$ ). 
6.4. The classification results above are based on the following reduction theorem. Let $V=\left(v_{1}, \ldots, v_{k}\right)$ be a regular bracket generating distribution on $M^{n}$. Assume that $k \leq n-2$ and $\operatorname{dim}[V, V]=\operatorname{dim} V+1$ (see 5.2). Then $V$ is equivalent to a distribution of the form

$$
\left(v_{1}(x), v_{2}(x), \frac{\partial}{\partial y_{1}}, \ldots, \frac{\partial}{\partial y_{k-2}}\right),
$$

where $x=\left(x_{1}, \ldots, x_{n-k+2}\right), v_{i}(x)$ are vector fields of the form $\sum a_{i j}(x) \frac{\partial}{\partial x_{j}}$.

6.5. The Goursat condition. Given a module $V$ of vector fields define a sequence of modules $V^{(1)}, V^{(2)}, \ldots$, where $V^{(1)}=V, V^{(j+1)}=\left[V^{(j)}, V^{(j)}\right]$. Note that in the general case this sequence of modules differs from the sequence $V_{1}$, $V_{2}, \ldots$ (see section 5). A distribution $V$ on $M^{n}$ satisfies the Goursat condition if $\operatorname{dim} V^{(j+1)}(p)=\min \left(n, \operatorname{dim} V^{(j)}(p)+1\right)$ for all points $p \in M^{n}$. The normal forms given in section 6.1 correspond to the Goursat normal forms in terms of Pfaffian systems (see Goursat (1923)). The results of section 6.1 do not follow from the Goursat results since the Goursat normal forms fail for "exceptional" distributions satisfying the Goursat condition (see, for example, Kumpera and Ruiz (1982)). If $(n-k) \geq 4$ then there is no general relation between regular distributions with the minimal growth vector $(k, k+1, k+2, \ldots, n)$ and $k$-distributions satisfying the Goursat condition (there are regular distributions with the minimal growth vector which do not satisfy the Goursat condition, and there are non-regular distributions which satisfy the Goursat condition). If $n-k=3$ then every regular distribution with the minimal growth vector satisfies the Goursat condition, but the converse is not true. If $n-k \leq 2$ then a distribution satisfies the Goursat condition if and only if it is a regular distribution with the minimal growth vector. Classification results for distributions satisfying the Goursat condition can be found in the work by A. Kumpera and C. Ruiz (1982).

\section{Singularities of fields of hyperplanes on $M^{2 k+1}, k \geq 2$}

7.0. First occurring singularities were studied by J. Martinet (1970). Classification of degenerations of codimension $\leq 4$ was obtained by the author (1989, 1992).

7.1. Let $W=(\omega)$ be a generic $2 k$-distribution on $M^{2 k+1}$. There exists a subset $R \subset W$, of codimension $\geq 4$, such that the germ of $W$ at any point of $M^{2 k+1}-R$ is equivalent to one and only one of the germs $(2.1),(7.1),(7.2),(7.3)$, where (7.1)-(7.3) are the following germs:

$$
\begin{gathered}
\left(d y_{1}+x_{2} d y_{2}+\ldots+x_{k} d y_{k}+x_{1}^{2} d z\right) \\
\left(d y_{1}+x_{2} d y_{2}+\ldots+x_{k} d y_{k}+\left(x_{1} y_{2}+x_{1}^{2} z\right) d z\right) \\
\left(d y_{1}+x_{2} d y_{2}+\ldots+x_{k} d y_{k}+\left(x_{1} y_{2}+x_{1}^{3} / 3+x_{1} z^{2}\right) d z\right) .
\end{gathered}
$$

Normal form (2.1) holds at generic points of $M^{2 k+1}$, normal form (7.1) at generic points of a stratified codimension 1 submanifold $S \subset M^{2 k+1}$, and normal 
forms (7.2) and (7.3) at generic points of a stratified codimension 2 submanifold $S_{1} \subset S$ (the codimension of $S_{1}$ in $M^{2 k+1}$ equals 3 ).

7.2. Denote by $S$ the set of points at which the form $\omega \wedge(d \omega)^{k}$ vanishes. The germ at $\alpha \in M^{2 k+1}$ of $W$ is equivalent to (2.1) if and only if $\alpha \notin S$. The germ is equivalent to (7.1) if and only if the following conditions hold true:

a) $\alpha \in S$;

b) $j_{\alpha}^{1}\left(\omega \wedge(d \omega)^{k}\right) \neq 0$

c) $\left.\left(\omega \wedge(d \omega)^{k-1}\right)\right|_{\alpha} \neq 0$

d) $\left.\operatorname{Ker}\left(\omega \wedge(d \omega)^{k-1}\right)\right|_{\alpha}+T_{\alpha} S=T_{\alpha} M^{2 k+1}$.

Note. If a point $\alpha$ satisfies b) and c) then $S$ is a smooth hypersurface near $\alpha$, and $\left.\operatorname{dim} \operatorname{Ker}\left(\omega \wedge(d \omega)^{k-1}\right)\right|_{\alpha}=2$.

7.3. Denote by $S_{1}$ a subset of $S$ consisting of points violating at least one of the conditions b)-d). The germ of $W$ at $\alpha \in S_{1}$ is equivalent to (7.2) or (7.3) if and only if $\alpha$ satisfies b) and c), and

e) $\left.\operatorname{Ker}\left(\omega \wedge(d \omega)^{k-1}\right)\right|_{\alpha}+T_{\alpha} S_{1}=T_{\alpha} S$.

Note. Near a point $\alpha$ satisfying b) and c), $S_{1}$ is a smooth codimension 2 submanifold of $S$.

7.4. To distinguish degenerations corresponding to (7.2) and (7.3) take a volume form $\mu$ on $S$ and introduce a vector field $X$ on $S$ by the relation $X\rfloor \mu=$ $\left.\left(\omega \wedge(d \omega)^{k-1}\right)\right|_{S}$ (we assume that the conditions b) and c) hold true). Any point $\alpha$ violating d) is a singular point of $X$; the spectrum of $X$ at $\alpha$ consists of $(n-3)$ zero eigenvalues and two more eigenvalues $\lambda_{1}$ and $\lambda_{2}$ whose sum is zero. Therefore only the following cases are possible: $\lambda_{1}$ and $\lambda_{2}$ are real non-zero numbers (the hyperbolic case), $\lambda_{1}$ and $\lambda_{2}$ are pure imaginary numbers (the elliptic case), and the parabolic case where both $\lambda_{1}$ and $\lambda_{2}$ are zero. The condition e) is equivalent to the condition $\lambda_{1} \lambda_{2} \neq 0$, so it excludes the parabolic case. Normal form (7.2) corresponds to the hyperbolic case, and normal form (7.3) to the elliptic one.

\section{Singularities of fields of planes on $M^{3}$}

8.0. First occurring singularities were studied by J. Martinet (1970). A numerical modulus corresponding to point singularities was found by B. Jakubczyk and F. Przytycki (1979). Classification was completed by the author (1989, 1992).

8.1. The germ at any point of a generic 2-distribution $W=(\omega)$ on $M^{3}$ is equivalent to one and only one of the germs $(8.1),(8.2),(8.3),(8.4)$, where (8.1)-(8.4) are the following germs:

$$
\begin{gathered}
(d z+x d y), \\
\left(d z+x^{2} d y\right), \\
\left(d y+\left(x y+x^{2} z+b x^{3} z^{2}\right) d z\right),
\end{gathered}
$$




$$
\left(d y+\left(x y+x^{3} / 3+x z^{2}+b x^{3} z^{2}\right) d z\right)
$$

where $b$ is an invariant distinguishing non-equivalent germs. Normal form (8.1) holds at generic points of $M^{3},(8.2)$ at generic points of a hypersurface $S \subset M^{3}$, and (8.3), (8.4) at isolated points of $S$.

8.2. The germ is equivalent to (8.1) if and only if

a) $\left.(\omega \wedge d \omega)\right|_{\alpha} \neq 0$.

If the 2-distribution is given as a 2-module $V=\left(v_{1}, v_{2}\right)$ of vector fields then this condition is equivalent to the condition $\operatorname{span}\left(v_{1}(\alpha), v_{2}(\alpha),\left[v_{1}, v_{2}\right](\alpha)\right)=$ $T_{\alpha} M^{3}$

8.3. A generic 2-distribution satisfies the condition

b) $j_{\alpha}^{1}(\omega \wedge d \omega) \neq 0$ for any $\alpha \in M^{3}$.

This condition implies that the set $S$ consisting of points violating a) is a smooth surface. The germ at $\alpha \in S$ is equivalent to (8.2) if and only if b) holds, and

c) $\left.\operatorname{Ker} \omega\right|_{\alpha} \neq T_{\alpha} S$.

8.4. Assume that b) holds. Take a volume form $\mu$ on $S$ and define a vector field $v$ on $S$ by the relation $X\rfloor \mu=\left.\omega\right|_{S}$. Any point $\alpha \in S$ violating c) is a singular point of $X$, the sum of the eigenvalues $\lambda_{1}$ and $\lambda_{2}$ of $X$ at $\alpha$ is 0 . The germ at $\alpha \in S$ is equivalent to (8.3) (resp. (8.4)) if and only if b) holds, c) is violated, and $\lambda_{1}$ and $\lambda_{2}$ are real non-zero (resp. pure imaginary) numbers.

\section{Singularities of fields of hyperplanes on $M^{2 k}$}

9.0. It was proved by J. Martinet (1970) that first occurring degenerations have codimension 3. This means that if $V$ is a generic $(2 k-1)$-distribution on $M^{2 k}$ then the set $S$ consisting of points at which the germ of $V$ is not equivalent to the Darboux-Pfaff model (2.2) is a stratified submanifold of $M^{n}$ of codimension 3. Moduli corresponding to the first occurring singularities (germs at points of $S$ ) were found by P. Mormul (1988). Classification results for germs at points of $S$ were obtained in the author's works $(1988,1992)$.

9.1. For a generic field of hyperplanes $W=(\omega)$ on $M^{2 k}, k \geq 3$ (resp. $k=2$ ) there exists a subset $R \subset M^{2 k}$, of codimension $\geq 4$, such that the germ of $W$ at any point $\alpha \in M^{2 k}-R$ is equivalent to one of the germs (2.2), (9.1) or (9.2) (resp. (2.2), (9.3), (9.4)), where (9.1)-(9.4) are the following germs:

$$
\begin{gathered}
\left(d y_{1}+x_{2} d y_{2}+\ldots+x_{k-1} d y_{k-1}+x_{1} y_{k}\left(1+\lambda+x_{2}\right) d x_{k}+x_{1} x_{k} d y_{k}\right), \\
\quad\left(d y_{1}+x_{2} d y_{2}+\ldots+x_{k-1} d y_{k-1}\right. \\
\left.\quad+\left(x_{1} x_{k}\left(2 \lambda+x_{2}\right)-x_{1} y_{k}\right) d x_{k}+\left(x_{1} x_{k}+x_{1} y_{k}\left(2 \lambda+x_{2}\right)\right) d y_{k}\right), \\
\left(d y+(1+\lambda+y) x v d u+x u d v+\nu(y) x^{2} u v^{2} d u\right), \\
\left(d y+(x u(2 \lambda+y)-x v) d u+(x u+x v(2 \lambda+y)) d v+\nu(y) x^{2} u v^{2} d u\right) .
\end{gathered}
$$


Here $\lambda$ is a real parameter distinguishing non-equivalent germs, $\lambda \in(0,1)$ for normal forms (9.1) and (9.3), $\lambda>0$ for normal forms (9.2) and (9.4). The families (9.3) and (9.4) are parametrized also by an arbitrary function in one variable $\nu(y)$; this function is also an invariant distinguishing non-equivalent germs.

Normal form (2.2) holds at generic points of $M^{2 k}$, the other normal forms at generic points of a codimension 3 stratified submanifold $S \subset M^{2 k}$.

9.2. The germ at $\alpha \in M^{2 k}$ is equivalent to (2.2) if and only if

a) $\left.\left(\omega \wedge(d \omega)^{k-1}\right)\right|_{\alpha} \neq 0$.

If $k=2$ then this condition can be easily formulated in terms of a 3-module $V$ of vector fields defining the 3-distribution: $[V, V](\alpha)=T_{\alpha} M^{2 k}$ (see 5.2).

9.3. Take a volume form $\mu$ on $M^{2 k}$ and define a vector field $X$ on $M^{2 k}$ by the relation $X\rfloor \mu=\omega \wedge(d \omega)^{k-1}$. Every singular point of $W$ (a point violating a)) is a singular point of $X$. Let $S$ be the set of singular points of $W$. Assume that $\alpha \in S$ and

b) $\left.\left(\omega \wedge(d \omega)^{k-2}\right)\right|_{\alpha} \neq 0$.

Then one can prove that the spectrum of $X$ at $\alpha$ consists of $(2 k-3)$ zero eigenvalues, and the sum of the other eigenvalues $\lambda_{1}, \lambda_{2}$ and $\lambda_{3}$ is zero. Normal form (9.1) (resp. (9.2)) holds at points $\alpha \in S$ satisfying b) and some other genericity conditions; one of them is as follows: $\lambda_{1}, \lambda_{2}$ and $\lambda_{3}$ are real non-zero numbers (resp. $\lambda_{1,2}=a \pm i b$, where $a \neq 0, b \neq 0$, and $\lambda_{3}$ is a real non-zero number, up to numbering).

9.4. Reduction to (9.1) and (9.3) is possible by a $C^{r}$-transformation, for any $r<\infty$, but, in general, a germ cannot be reduced to $(9.1)$ or $(9.3)$ by a $C^{\infty}$ transformation. This does not concern normal forms (9.2) and (9.4) (reduction to the latter normal forms is possible by a $C^{\infty}$ transformation).

\section{Singularities of 2-distributions on $M^{4}$ (Zhitomirskiŭ (1990a))}

10.1. Let $V=\left(v_{1}, v_{2}\right)$ be a generic 2-distribution on $M^{4}$. There exist two 2-dimensional surfaces $S_{1} \subset M^{4}, S_{2} \subset M^{4}$, and a curve $R \subset S_{2}$ such that

- the intersection of $S_{1}$ and $S_{2}$ is a smooth curve $L\left(S_{1}\right.$ and $S_{2}$ are not transversal);

- the germ of $V$ at a point $\alpha \notin S_{1} \cup S_{2}$ is equivalent to the germ (2.3);

- the germ of $V$ at a point $\alpha \in S_{1}-L$ is equivalent to the germ (5.1);

- the germ of $V$ at a point $\alpha \in\left(S_{2}-(L \cup R)\right)$ is equivalent to one of the germs (5.2), (5.3).

10.2. The surface $S_{1}$ consists of points at which $\operatorname{dim} V_{2}=2$, and the surface $S_{2}$ of points at which $\operatorname{dim}_{3}<4$ (see notations in section 5). The curve $R$ consists of points at which the distribution $V$ is not transversal to $S_{2}$. This curve is transversal to $L$. The transversality of $V$ and $S_{1}$ holds at any point $\alpha \in S_{1}-L$. 
The growth vector at points of $S_{1}-L$ equals $(2,2,4)$, and at points of $S_{2}-(L \cup R)$ it is $(2,3,3,4)$.

10.3. The germ of any $V$ at a point $\alpha \in M^{n}$ is equivalent to (2.3) (resp. (5.1)) if and only if the growth vector at $\alpha$ equals $(2,3,4)$ (resp. $(2,2,4)$ ).

10.4. Take a local volume form $\mu$ on $M^{4}$ and define a 1-form $\omega$ by the equation $\omega(Y)=\mu\left(v_{1}, v_{2},\left[v_{1}, v_{2}\right], Y\right)$ (for any local vector field $Y$ ). Now define a vector field $Z$ by the relation $Z\rfloor \mu=\omega \wedge d \omega$. Every singular point of $V$ (a point at which the growth vector is not equal to $(2,3,4))$ is a singular point of $Z$. If $\operatorname{dim}_{3}(\alpha)<4$ then the spectrum of $Z$ at $\alpha$ consists of two zero eigenvalues and two more eigenvalues $\lambda_{1}, \lambda_{2}$ whose sum is zero. The germ at $\alpha$ is equivalent to (5.2) (resp. (5.3)) if and only if the growth vector at $\alpha$ equals $(2,3,4)$ and the eigenvalues $\lambda_{1}$ and $\lambda_{2}$ are real non-zero (resp. pure imaginary).

11. Stable germs. A complete description of stable germs of 2-distributions on $M^{3}$ follows from the results by B. Jakubczyk and F. Przytycki (1979). The other results of this section were obtained in the author's works $(1988,1990 \mathrm{a}$, 1992).

11.1. A germ of a distribution $D$ at a point $\alpha \in M$ is called stable if for any neighbourhood $U$ of $\alpha$ there exists a neighbourhood $A$ of $D$ (in the Whitney topology) such that for any distribution $\tilde{D} \in A$ there exists a point $\tilde{\alpha} \in U$ such that the germ of $\tilde{D}$ at $\tilde{\alpha}$ is equivalent to the germ of $D$ at $\alpha$.

11.2. A complete description of stable germs of $k$-distributions on $M^{n}$ is as follows. There are no stable germs if $(k, n)$ is not a "classical" pair, i.e. if $2 \leq k \leq n-2$ and $(k, n) \neq(2,4)$ (see section 3 ). Every stable germ of a $2 k$ distribution on $M^{2 k+1}, k \geq 2$, is equivalent to one of the germs (2.1), (7.1), (7.2) and (7.3) (Zhitomirskii (1992)). Every stable germ of a 2-distribution on $M^{3}$ is equivalent to one of the germs (8.1), (8.2) (Jakubczyk and Przytycki (1979)). Every stable germ of a $(2 k-1)$-distribution in $M^{2 k}, k \geq 3$, is equivalent to one of the germs (2.2), (9.1), (9.2) (the existence of the invariant $\lambda$ in normal forms (9.1), (9.2) is not in conflict with stability; Zhitomirskiu $(1988,1992))$. Every stable germ of a 3 -distribution on $M^{4}$ is equivalent to the germ $\left(d y_{1}+x_{2} d y_{2}\right)$ (the germ of the standard quasi-contact structure) (Zhitomirskii (1988)). Every stable germ of a 2-distribution on $R^{4}$ is equivalent to one of the germs (2.3), (5.1), (5.2) and (5.3) (Zhitomirskil (1992)).

\section{Finitely determined germs (Zhitomirskii (1992))}

12.1. A germ $\left(v_{1}, \ldots, v_{k}\right)$ is called $k$-determined if it is equivalent to any germ $\left(\tilde{v}_{1}, \ldots, \tilde{v}_{k}\right)$ such that $j^{k} \tilde{v}_{i}=j^{k} v_{i}, \quad i=1, \ldots, k$. A germ is called finitely determined if it is $k$-determined for some $k<\infty$.

12.2. Every stable germ of a $k$-distribution on $M^{n}$ is finitely determined. Namely, germs (2.1), (2.2) and (8.1) are 1-determined, germs (2.3), (5.1), (7.1) and 
(8.2) are 2-determined, germs (5.2), (5.3), (7.2), (7.3), and (9.2) are 3-determined. Germ (9.1) is 3-determined with respect to $C^{r}$-equivalence, for any $r<\infty$ (see $9.4)$.

12.3. Every germ of a generic field of planes on $M^{3}$ is finitely determined: germ (8.1) is 1-determined, (8.2) is 2-determined, (8.3) and (8.4) are 5-determined.

12.4. Let $W=(\omega)$ be a generic field of hyperplanes on $M^{2 k+1}, k \geq 2$. At some isolated singular points $\alpha \in S$ (see definition of $S$ in 7.2) we encounter the degeneration $\left.\left(\left.\omega\right|_{S}\right)\right|_{\alpha}=0$ (i.e. $\left.\operatorname{Ker} \omega\right|_{\alpha}=T_{\alpha} S$ ). If $\alpha$ is such a point then the germ of $W$ at $\alpha$ is not stable, but it is 5-determined. The normal form corresponding to this degeneracy contains $k$ moduli, it can be found in (Zhitomirskiu (1992)).

12.5. Any finitely determined germ of a generic field of planes on $M^{2 k}, k \geq 2$, is stable. The same is true for finitely determined germs of generic 2-distributions on $M^{4}$. Finitely determined non-stable germs of generic $k$-distributions on $M^{n}$ exist only in the case where $n$ is odd and $k=n-1$ (germs (8.3), (8.4) in the 3 -dimensional case, and the degeneration described in 12.4).

\section{References}

R. L. Bryant (1994), Smooth normal form for generic germs of 2-distributions on 5-manifolds, a letter to the author.

R. L. Bryant and L. Hsu (1993), Rigidity of integral curves of rank 2 distributions, Invent. Math. 114, 435-461.

F. Engel (1889), Zur Invariantentheorie der Systeme von Pfaffschen Gleichungen, Berichte Verhandl. Königl. Sachsischen Gesell. Wiss. Math.-Phys. Kl. 41.

E. Goursat (1923), Leçons sur le Problème de Pfaff, Hermann, Paris.

B. Jakubczyk and F. Przytycki (1979), On J. Martinet's conjecture, Bull. Polish Acad. Sci. Math. 27.

- (1984), Singularities of $k$-tuples of vector fields, Dissertationes Math. (Rozprawy Mat.) 213.

A. Kumpera and C. Ruiz (1982), Sur l'équivalence locale des systèmes de Pfaff en drapeau, in: Monge-Ampère Equations and Related Topics, Inst. Alta Mat., Rome, 201-248.

W. Liu and H. J. Sussmann (1994), Shortest paths for sub-Riemannian metrics of rank-2 distributions, preprint, Rutgers University.

J. Martinet (1970), Sur les singularités des formes différentielles, Ann. Inst. Fourier (Grenoble) $20(1), 95-178$.

R. Montgomery (1995), Abnormal minimizers, SIAM J. Control Optim., to appear.

P. Mormul (1988), Singularities of triples of vector fields on $R^{4}$ : the focusing stratum, Studia Math. 91, 241-273.

A. M. Vershik and V. Ya. Gershkovich (1988), An estimate of the functional dimension for the space of orbits of germs of generic distributions, Math. Notes 44 (5), 806-810.

-, - (1989), A bundle of nilpotent Lie algebras over a nonholomorphic manifold (nilpotentization), Zap. Nauchn. Sem. Leningrad. Otdel. Mat. Inst. Steklov. 172 (10), 21-40 (in Russian).

M. Zhitomirskiǔ (1988), Singularities and normal forms of even-dimensional Pfaff equations, Russian Math. Surveys 43 (5), 266-267. 
M. Zhitomirskiǔ (1989), Singularities and normal forms of odd-dimensional Pfaff equations, Functional Anal. Appl. 23 (1), 59-61.

- (1990), Normal forms of germs of distributions with a fixed growth vector, Algebra i Anal. 2 (5), 125-149 (in Russian).

- (1990a), Normal forms of germs of 2-dimensional distributions on $R^{4}$, Functional Anal. Appl. 24 (2), 150-152.

- (1991), Normal forms of germs of smooth distributions, Math. Notes 49 (2), 139-144.

- (1992), Typical Singularities of Differential 1-Forms and Pfaffian Equations, Transl. Math. Monographs 113, Amer. Math. Soc., Providence, 1992. 